\title{
Selection and Characterization of a Novel DNA
Aptamer, Apt-07S Specific to Hepatocellular Carcinoma Cells
}

This article was published in the following Dove Press journal: Drug Design, Development and Therapy

\author{
Xiao-Xia Yu $\mathbb{D}^{1, *}$ \\ $\mathrm{Ke}-\mathrm{Li} \mathrm{Ge}{ }^{2, *}$ \\ Ning Liu $^{3}$ \\ Jin-Yu Zhang' \\ Mei-Lan Xue' \\ Yin-Lin Ge' \\ 'Department of Biochemistry and \\ Molecular Biology, Basic Medical College, \\ Qingdao University, Qingdao, Shandong \\ Province 26607I, People's Republic of \\ China; ${ }^{2}$ Integrative Medicine Research \\ Center, Medical College, Qingdao \\ University, Qingdao 26602I, Shandong \\ Province, People's Republic of China; \\ ${ }^{3}$ Department of Dermatology, Qingdao \\ Municipal Hospital, Qingdao 26607I, \\ Shandong Province, People's Republic of \\ China
}

*These authors contributed equally to this work
Correspondence: Yin-Lin Ge Department of Biochemistry and Molecular Biology, Basic Medical College, Qingdao University, 308 Ningxia Road, Qingdao 26607I, People's Republic of China

Email geyinlin@।26.com
Background: The efficacy of traditional therapeutic methods for liver cancer is unsatisfying because of the poor targeting, and inefficient drug delivery system. A recent study has proven that aptamers, developed through cell-SELEX, could specifically recognize cancer cells and show great potential in the development of a delivery system for anticancer drugs.

Purpose: To develop a hepatocellular carcinoma specific aptamer using two kinds of hepatocellular carcinoma cell lines, $\mathrm{HepG}_{2}$ and SMMC-7721, as double targets and a normal hepatocyte, L02, as a negative control cell.

Methods: Hepatocellular carcinoma specific aptamer was developed via cell-SELEX. The enrichment of the library was monitored by flow cytometric analysis. The specificity, affinity, and distribution of the candidate aptamer were explored. Further study was carried to assess its potential in drug delivery.

Results: The library was enriched after 14 rounds of screening. Candidate aptamer Apt-07S can recognize four kinds of hepatocellular carcinoma cells and show little cell-binding ability to normal cells and four cell lines of different cancer types, revealing a high specificity of Apt-07S. Confocal imaging showed that Apt-07S distributed both on the surface and in the cytoplasm of the two target cells. Moreover, an anti-sense nucleotide to gene Plk1 (ASOPlk1) was connected at the 3' end of Apt-07S to form an integrated molecule (Apt-07S-ASOPlk1); the functional analysis indicated that the structure of Apt-07S may help ASO-Plk1 enter the cancer cells.

Conclusion: The study indicates that Apt-07S can specifically target HCC and may have potential in the delivery of anticancer drugs.

Keywords: aptamer, cell-SELEX, hepatocellular carcinoma, double target

\section{Introduction}

Liver cancer, usually known as "king of cancer", is one of the most common malignant tumors in the clinic. The incidence of liver cancer is the fifth-highest among malignant tumors, and the mortality rate ranks second worldwide in 2018. ${ }^{1}$ Hepatocellular carcinoma (HCC) accounts for about $90 \%$ of all cases of primary liver cancer. ${ }^{2}$ In most cases, patients cannot be diagnosed at an early stage due to the lack of obvious symptoms and accurate diagnostic methods. Surgical resection and non-surgical treatments, e.g. locoregional therapies, were once the main methods in treating cases with advanced HCC; however, the five-year survival rate of patients remained poor as a result of the high recurrence rate or metastasis rate. ${ }^{3}$ In recent years, molecular-targeted drugs, such as sorafenib, ${ }^{4}$ have been widely used in the treatment of advanced HCC. 
However, the therapeutic efficacy is unsatisfying because the survival extension is less than 3 months, and is accompanied by serious side effects. ${ }^{5}$ Thus, the development of early detection methods and other effective targeted drugs would bring new breakthroughs in the treatment of hepatocellular carcinoma.

Aptamers are short single-strand DNA or RNA oligonucleotides that can specifically bind to a target, such as a metal ion, antibiotic, protein, or cell, with high affinity and stability. Aptamers are selected from a random oligonucleotide library in vitro by a technique named Systematic Evolution of Ligands by Exponential enrichment (SELEX). ${ }^{6,7}$ Cell-SELEX, ${ }^{8}$ which is based on SELEX, utilizes the whole cell as targets during the process of aptamer selection. With cell-SELEX, aptamers can be isolated without prior knowledge of the cancerspecific biomarker, thus making it possible to discover more potential biomarkers and cancer-specific aptamers for cancer cells. ${ }^{9-13}$ Compared with conventional antibodies, aptamers are more easily synthesized and modified, with higher stability and reproducibility in different batches, and their lower immunogenicity ${ }^{14}$ gives them great potential in the recognition of cancer cells ${ }^{15-18}$ and specific delivery of anticancer drugs. ${ }^{19-21}$

To date, several aptamers have been developed against human-derived hepatocellular cell lines, for example, $\mathrm{HepG}_{2},{ }^{11,16,22-25}$ HCCLM9 ${ }^{26}$ and ${ }^{2} H 86^{27}$ were verified to recognize their targets specifically in vitro. Some other aptamers were applied to conjugate with anticancer drug doxorubicin (Dox) or oligonucleotides for targeting therapy as delivery agents. ${ }^{23,28,29}$ To sum up, cell line $\mathrm{HepG}_{2}$ has been widely used as the target cell during the selection, verification, and application of aptamers in vitro. However, according to the American Tissue Culture Collection (ATCC), the poor tumorigenicity of $\mathrm{HepG}_{2}$ in nude mice greatly limits its application in experiments in vivo. By contrast, cell line SMMC-7721, derived from a 50-year-old Chinese male, has been increasingly used as a model to study hepatocellular carcinoma in vivo due to the high xenotransplantation. ${ }^{30-32}$ Given that, we applied HepG $_{2}$ and SMMC-7721 as double targets of the positive selection during cell-SELEX in order to develop an aptamer targeting a wide range of hepatocellular cell lines that would be well applied both in vitro and in vivo. In addition, a counter-selection was applied by using the normal hepatocyte, L02, as a negative control to isolate aptamer binding to target cells but no control cells.
We also prepared an integrated ssDNA (Apt-07S-ASOPlk1) with a 20 nt anti-sense oligonucleotide (ASODN) directed against gene Plk1. Plk1, polo-like kinase 1, is a cell-proliferation associated gene which is usually overexpressed in cancer cells, while ASODNs are short oligonucleotides that can lead to gene silencing by the RNase $\mathrm{H}$ pathway. Thus, the uptake of ASODNs against Plk1 (ASOPlk1) may lead to growth inhibition of cancer cells. ${ }^{33} \mathrm{We}$ connected ASO-Plk1 with the selected aptamer Apt-07S to make an integrated ssDNA (Apt-07S-ASO-Plk1). Compared with ASO-Plk1, increasing the inhibitory rate of Apt-07S-ASO-Plk1 to $\mathrm{HepG}_{2}$ might indicate the potential of Apt-07S in the delivery of anticancer drugs into cancer cells.

\section{Materials and Methods Cell Lines and Reagents}

Ten human-derived cell lines were used in this study: $\mathrm{HepG}_{2}$ (hepatocellular carcinoma), MCF-7 (breast carcinoma), H460 (large cell lung carcinoma), MGC-803 (gastric carcinoma), SW480 (colon adenocarcinoma), HCC-LM3 (hepatocellular carcinoma), Huh.7 (hepatocellular carcinoma), SMMC-7721 (hepatocellular carcinoma), L02 (normal hepatocytes), and HBL-100 (normal breast cell line incorporated with SV40 virus gene). Four cell lines $\left(\mathrm{HepG}_{2}, \mathrm{MCF}-7\right.$, H460, and MGC-803) were provided by the American Type of Culture Collection (ATCC). SW480, Huh.7, SMMC-7721, L02, and HBL100 were provided by Shanghai Cell Bank of the Chinese Academy of Sciences. HCC-LM3 was provided by the China Center for Type Culture Collection in Wuhan. All these cell lines were cultured in Dulbecco's modified Eagle's medium with high glucose (H-DMEM, Gibco) supplemented with 10\% fetal bovine serum (FBS, Gibco), 100 units/mL penicillin and $100 \mu \mathrm{g} / \mathrm{mL}$ streptomycin. All cells were incubated in an incubator at $37^{\circ} \mathrm{C}$ with $5 \% \mathrm{CO}_{2}$ atmosphere.

$\mathrm{HepG}_{2}$ and SMMC-7721 cells were both selected as positive target cells and were used alternately every three rounds. L-02 cells were used as negative control cells from the 4th round. Dulbecco's phosphate-buffered saline (PBS, Gibco) supplemented with $1 \mathrm{mM} \mathrm{MgCl}_{2}$ was used as washing buffer before or after the incubation. During the SELEX, 0.1 $\mu \mathrm{g} / \mu \mathrm{L}$ yeast tRNA (ytRNA, Sigma), and $0.1 \mu \mathrm{g} / \mu \mathrm{L}$ salmon sperm DNA (wDNA, Sigma) were added into the washing buffer to reduce the background binding. 


\section{SELEX Library and Primers}

The initial library and primers were synthesized by Sangon Biotech (Shanghai, China), and the sequences were as follows:

Gp30: 5'-GCAATGGTACGGTACTGTC (30N) AATC AGTGCACGCTACTTTGCTAA-3'

Plong-1 tgtc (Forward primer 1): 5' -GCAATGGT ACGGTACTGTC-3'

P11-tgatt (Reverse primer 1): 5'-TTAGCAAAGTAG CGTGCACTGATT-3'

Pstemloop-tgatt (Reverse primer 2): 5'-GCTAAGCG GGTGGGACTTCCTAGTCCCACCCGCTTAGCAAAGTAGCGTGCACTGATT-3'.

FAM- Plong-1 tgtc (Forward primer 2): 5'-FAMGCAATGGTACGGTACTGTC-3'

The initial library Gp30 consists of a random sequence of 30 nucleotides in the middle and a fixed sequence at both ends. Plong-1 tgtc and Pstemloop-tgatt were used for the amplification of the single-stranded DNA (ssDNA) library by unequal length strand PCR. ${ }^{34}$ Primer Plong-1 tgtc and P11-tgatt were used to obtain the double-stranded deoxyribonucleic acid (dsDNA) for monoclonal sequencing. FAM-labeled Plong-1 tgtc was used to prepare FAMlabeled ssDNA library, which was used to monitor the enrichment of the ssDNA library.

\section{SELEX Procedure}

Approximately 6000 target cells $\left(\mathrm{HepG}_{2}\right.$ or $\mathrm{SMMC}-7721$ cells, alternatively used every two rounds) were inoculated into 96-well plates and cultured overnight. The cells were washed twice before the incubation with the library. The library (2000 pmol for the initial round, $200 \mathrm{pmol}$ for the second round, and decreasing for subsequent rounds) was dissolved in washing buffer, denatured at $95^{\circ} \mathrm{C}$ for $5 \mathrm{~min}$ and rapidly cooled on ice for $5 \mathrm{~min}$, then ytRNA and wDNA were added into the buffer. The final incubation system consisted of a $1 \times \mathrm{PBS}-1 \mathrm{mM} \mathrm{MgCl}_{2}-0.1 \mu \mathrm{g} / \mu \mathrm{L}$ ytRNA- $0.1 \mu \mathrm{g} / \mu \mathrm{L}$ wDNA-X pmol ssDNA library. After incubation for $30 \mathrm{~min}$ at $4{ }^{\circ} \mathrm{C}$, the cells were gently washed with washing buffer 3 times (gradually increased in subsequent rounds, from 3 to 5 times) to remove unbound ssDNA. Then, the cells with bound ssDNA were eluted by double-distilled water (DDW) at $95^{\circ} \mathrm{C}$. The eluent was heated at $95^{\circ} \mathrm{C}$ for $10 \mathrm{~min}$ to inactivate the DNase, and then used as templates to amplify the bound ssDNA by unequal length strand PCR with primer Plong-1 tgtc and Pstemloop-tgatt. The amplification and purification of the
ssDNA were carried out based on the protocol developed by $\mathrm{Li}^{34}$ Part of the purified ssDNA was input into SELEX as a subsequent library.

A counter selection was applied by the introduction of the negative control cell, L-02. From the 4th round, the subsequent library was firstly incubated with L-02 cells for 30 min at $4{ }^{\circ} \mathrm{C}$, followed by the supernatant collected and incubated with target cells.

The entire selection procedure would not be shut off until the ssDNA library was enriched. After several rounds of selection, the enriched library was then amplified into dsDNA with Plong-1 tgtc and P11-tgatt and cloned into a pMD-18T vector for monoclonal sequencing. The clones were sequenced by Sangon Biotech (Beijing, People's Republic of China). The secondary structure was plotted with RNA structure software (Version 6.2) and the structural homology was analyzed by MEME online software (http://meme.sdsc.edu/meme/cgi-bin/meme.cgi). Several candidate aptamers were selected based on the structure analysis, and then synthesized and labeled with FAM at $5^{\prime}$ end (Sangon Biotech, Shanghai, China).

\section{Flow Cytometric Analysis}

A flow cytometric assay was applied to monitor the enrichment of the ssDNA library or determine the specificity and the binding affinity of the candidate aptamers.

All the cell lines used in this study were adherent cells, and trypsin was used to detach cells. The suspended cells were washed with the washing buffer and counted. FAM-labeled ssDNA library (50 pmol) or FAM-labeled candidate aptamer $(100 \mathrm{pmol})$ was then incubated with $1-3 \times 10^{5}$ cells in a total volume of $200 \mu \mathrm{L}$ at $4^{\circ} \mathrm{C}$ for $30 \mathrm{~min}$. The cells were washed gently twice with the washing buffer. The fluorescence intensity on the cells was determined with a flow cytometer (BD Bioscience, San Jose, CA, USA) by counting 40,000 events. The FAM-labeled initial library Gp30 was used as a control.

The fluorescence intensity for the best candidate aptamer was the strongest during the cell-binding assay toward the target cells, and the binding affinity (Kd value) was evaluated by the equation $\mathrm{Y}=\mathrm{B}_{\max } \mathrm{X} /\left(\mathrm{K}_{\mathrm{d}}+\mathrm{X}\right)$ using the software GraphPad Prism 6.0. Several other human carcinoma cell lines or normal cells were subjected to the binding assay to determine the specificity of the best candidate aptamer in this study.

\section{Confocal Imaging}

Confocal imaging was carried out with an LSM710 confocal microscope (Zeiss, Jena, Germany) to observe the 
binding position of FAM-labeled aptamers on the cells. FAM-labeled aptamers were incubated with target cells $\left(\mathrm{HepG}_{2}\right.$ and SMMC-7721) and negative cell L02, respectively, at a concentration of $250 \mathrm{nM}$ for $30 \mathrm{~min}$ at $4^{\circ} \mathrm{C}$. The cells were then gently washed twice with washing buffer $\left(1 \times \mathrm{PBS}, 1 \mathrm{mM} \mathrm{MgCl}_{2}\right)$. After that, 4',6-diamidino-2-phenylindole (DAPI) was input into the buffer, and incubated for 10-30 $\mathrm{min}$ for the nucleus stain. Before the observation, five washes were necessary to remove the uncombined DAPI and reduce the background. All the incubation and washing operations should be performed in the dark to avoid fluorescence quenching.

\section{Analysis of Cell-Growth Inhibition Rate in vitro}

A 20nt ASODN (5'-CCCAGCCTTCCAGCTCCTTG-3') that specifically blocked the Plk1 gene was connected to the $3^{\prime}$ end of aptamer Apt-07S. An additional two bases, $g$ and a, were added between Apt-07S and ASODN-Plk1 to ensure the integrity of the secondary structure of Apt-07S. The three ssDNAs were modified with phosphorothioate to confer nuclease resistance and, therefore, enhance intracellular stability. The integrated ssDNA (Apt-07S-ASOPlk1) was incubated with the two target cells or control cell in 96-well plates at serial concentration gradient for 24 h. Then, a cell counting kit-8 (Vazyme, Nanjing, China) was used for the analysis of cell proliferation.

\section{Analysis of Western Blotting}

To identify whether there was a change in the expression of Protein Plk1 in cell line $\mathrm{HepG}_{2}$ and SMMC-7721 treated with the integrated ssDNA (Apt-07S-ASO-Plk1), Western blotting was carried out. After incubation with the integrated ssDNA (Apt-07S-ASO-Plk1) for $24 \mathrm{~h}$ at a concentration of $20 \mu \mathrm{M}$, cells were washed 2 times and the whole-cell protein sample was then extracted, quantified with the standards, and prepared by boiling in $0.1 \%$ SDS for $5 \mathrm{~min}$. Total protein $(100 \mu \mathrm{g})$ was separated by applying sodium dodecyl sulfate-polyacrylamide gel electrophoresis (SDS-PAGE) and transferred onto polyvinyl dine difluoride (PVDF). A block was performed by $5 \%$ non-fat milk, followed by the incubation with an antibody against PLK1 (dilution 1:500), and horseradish peroxidase (HRP) conjugated goat anti-rabbit IgG (dilution 1:1000). The antibodies were purchased from Abcam (England). Most of the reagents and facilities used during the process of sample preparation and SDS-PAGE were purchased from Bio-rad (America) and Solarbio (Beijing, China). The protein PLK1 was finally detected on the FUSION FX7 Spectra system (Viber, France).

\section{Statistical Analysis}

All experiments were repeated at least three times for each group. All values are expressed as means \pm SEM. Two-way ANOVA analysis was applied to test whether there were significant differences in cell viability among the groups treated differently at a series of concentration gradients. All histograms were drawn using GraphPad Prism 6.0.

\section{Results}

\section{The Enrichment of the ssDNA Library}

Flow cytometric analysis was applied to monitor the enrichment of the ssDNA library. The initial Gp30 library was directly labeled with FAM during the synthesis, and was chosen as the control. Several FAM-labeled subsequent ssDNA libraries (8th, 11th, and 14th rounds) were prepared with FAM-labeled primer Plong-1 tgtc and Stem loop primer Pstemloop-tgatt by unequal length strand PCR during the SELEX. It was observed that there was a gradual fluorescence intensity increase on the target cell $\mathrm{HepG}_{2}$ as the selection proceeded, but the same phenomenon was not observed in the negative cell L02 (Figure 1). The results indicated that the ssDNA library with high binding affinity had already enriched at the 14th round.

\section{Selection of Candidate Aptamers}

The enriched library was amplified into dsDNA and cloned to obtain monoclonal sequences; 100 random clones were finally sequenced and analyzed. There were four sequences that were repeated several times, Apt-31S (33nt, 5 times), Apt-07S (47nt, 3 times), Apt-8 (73nt, 3 times), and Apt-37S (31nt, 2 times). Five other sequences were selected based on the structure analysis and homology. In total, nine candidate aptamers were selected as candidate aptamers. Details of all nine candidate aptamers are shown in Table 1.

\section{Primary Screening of Candidate Aptamers}

All the nine candidate aptamers and Gp30 (the initial library) were labeled directly with FAM during the synthesis. Flow cytometry was first applied to determine the cell binding ability of candidate aptamers to $\mathrm{HepG}_{2}$, one of the two target cells, which was a primary 


\section{Target cell $\left(\mathrm{HepG}_{2}\right)$}

A

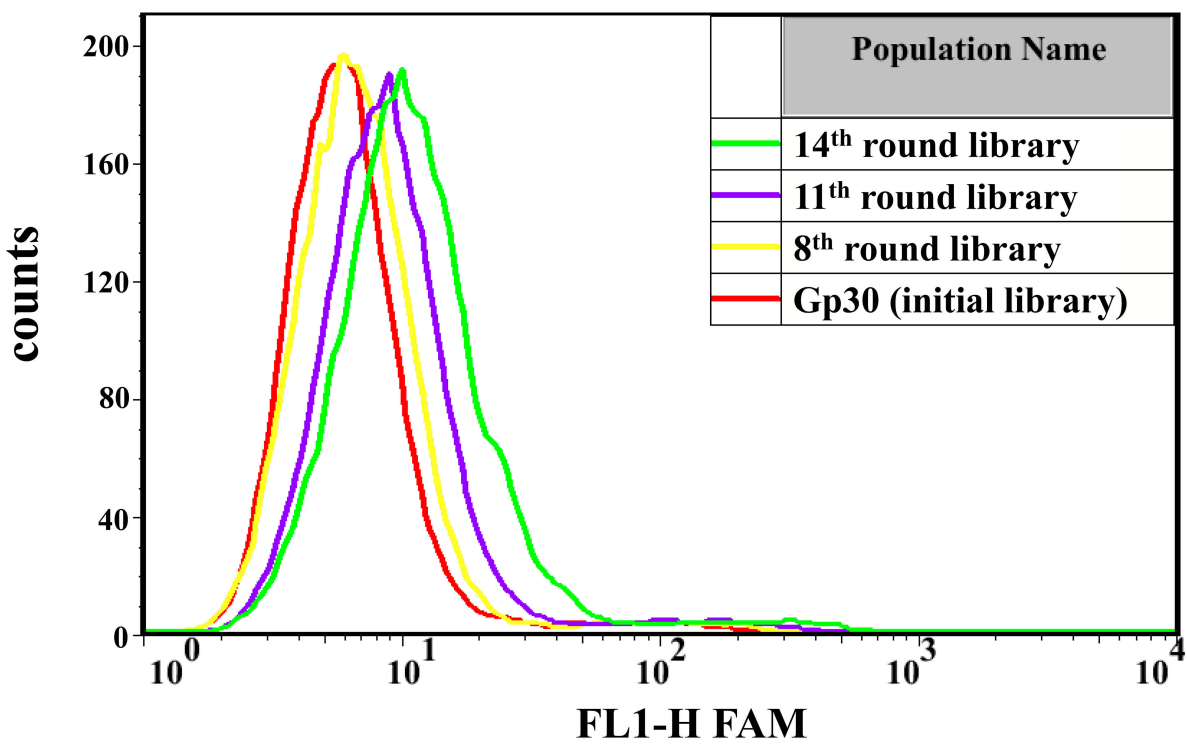

Control cell (L02)

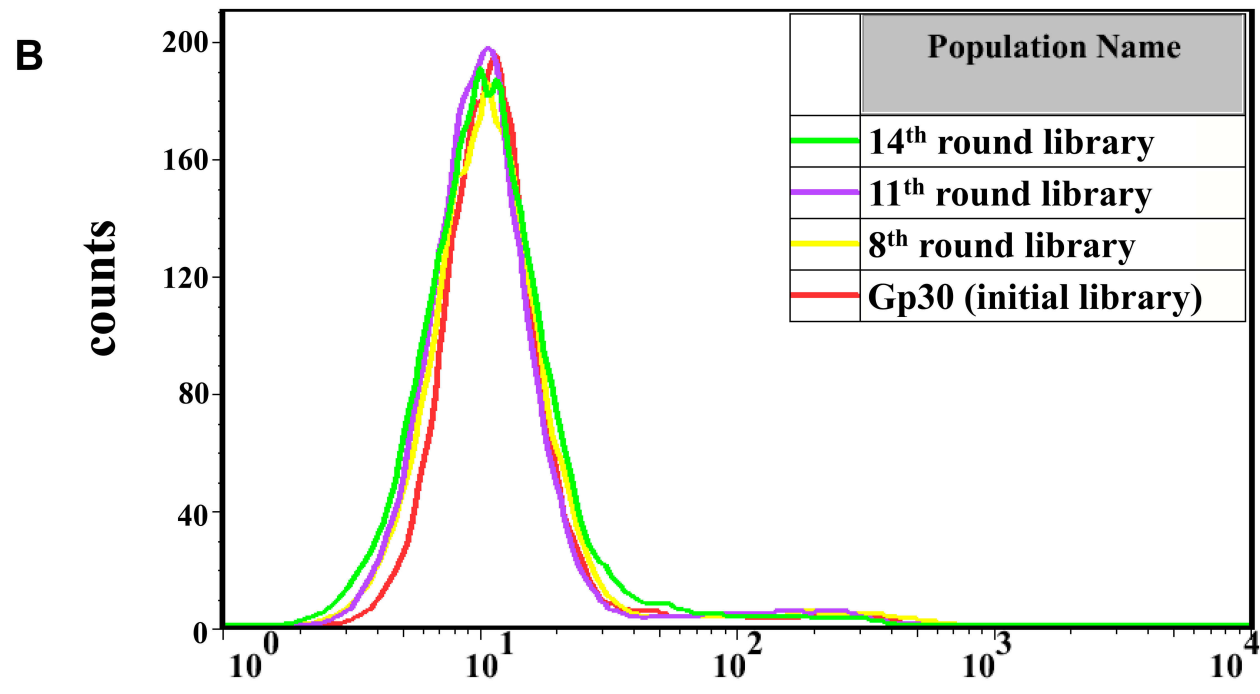

FL1-H FAM

Figure I Monitoring the enrichment of the ssDNA library by applying flow cytometry. (A) Fluorescence intensity analysis of the libraries binding to the target cell $\mathrm{HepG}_{2}$. (B) Fluorescenece intensity analysis of the libraries binding to the control cell L02. FAM-labeled Gp30 and ssDNA library (8th, II th, and I4th rounds) were, respectively, incubated with target cell $\mathrm{HepG}_{2}$ and counter cell L02, the final concentration of each input ssDNA library was $250 \mathrm{nM}$. The fluorescence intensity indicated the cell-binding ability of ssDNA libraries.

screening for the candidate aptamers. It is shown in Figure $2 \mathrm{~A}$ and $\mathrm{B}$ that the fluorescence intensity of Apt-07S and Apt-37S was the highest among the candidate aptamers; thus, they were promoted for further verification. Aptamer Apt-02, Apt-04, and Apt-08 did not perform well in the cell-binding assay for the insignificant increase in fluorescence intensity compared with Gp30. Additionally, there was no indication that the other three aptamers, Apt-09S, Apt-15, and Apt-32S, had stronger cell-binding ability to $\mathrm{HepG}_{2}$ than Gp30.
Unexpectedly, aptamer Apt-31S with a high frequency in monoclonal sequencing showed far lower binding ability to the target cell than the initial library, which is worth further discussion.

\section{Specificity and Binding Affinity of the Selected Aptamers}

Again, flow cytometry was employed to verify the specificity of aptamer Apt-07S and Apt-37S. As shown in Table 2 and Figure S1, it was observed that aptamer Apt- 
Table I The Sequences of Nine Candidate Aptamers

\begin{tabular}{|l|l|l|}
\hline Name & Sequence (5' to $\mathbf{3}^{\prime}$ ) & Length (nt) \\
\hline Apt-02 & GCAATGGTACGGTACTGTCGAGATTGGAGGCACGCGTACTACGGGTTTGAATCAGTGCACGCTACTTTGCTAA & 73 \\
Apt-04 & GCAATGGTACGGTACTGTCCGGGGCGTATCTTGGCATTAATCAGTGCACGCTACTTTGCTAA & 62 \\
Apt-07S & GTACTGTCAATTGGAAGTGGTGTTACGTTGTGTAGTCAAATCAGTGC & 47 \\
Apt-08 & GCAATGGTACGGTACTGTCGGGCTTGGGCGTCTTGTAGTCGTGGCTTTCAATCAGTGCACGCTACTTTGCTAA & 73 \\
Apt-09S & GGTACTGTCTATGGGAGATTAGACAAACGGATGGTGTTCAATCAGTGCACGCTACT & 56 \\
Apt-15 & GCAATGGTACGGTACTGTCACCATGTGGGCGTTTGTAAATCAGTGCACGCTACTTTGCTAA & 61 \\
Apt-3IS & GTCCGTATGCTTGATGTGACGTCACGGACGAGG & 33 \\
Apt-32S & GTACTGTCCGGATTCAGGTTCATCCCTTTTCCCGCAGAAATCAGTGC & 47 \\
Apt-37S & TGTTCAGCTCTAGGAGCTCACGCTCAGCTCA & 31 \\
\hline
\end{tabular}

37S showed cell-binding ability to the subtotal tested cell lines, not just to $\mathrm{HepG}_{2}$, implying poor cell specificity. By contrast, aptamer Apt-07S could specifically recognize two target cells $\left(\mathrm{HepG}_{2}\right.$ and SMMC-7721) but not negative cell L02, and showed certain binding ability to two other kinds of hepatocellular carcinoma cells, HCC-LM3 and Huh.7. In addition, it barely recognized the other three kinds of carcinoma cells, including MCF-7, H460, and

A

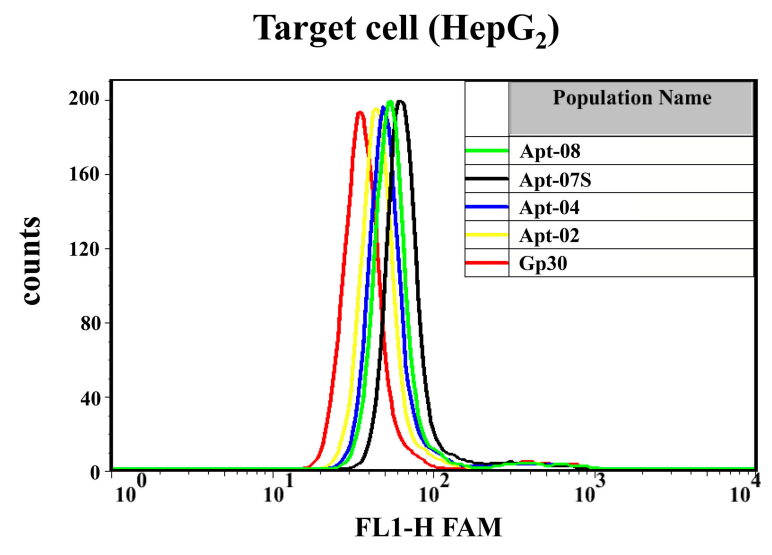

Control cell (L02)

C

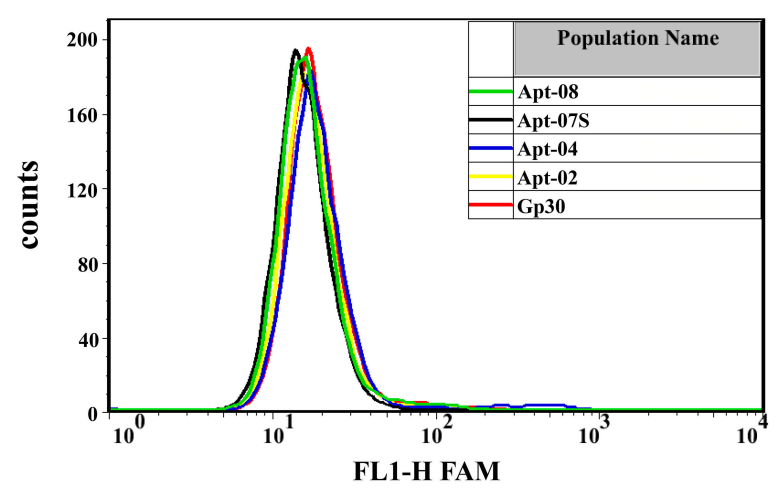

SW480, and normal breast cell line HBL-100, indicating that aptamer Apt-07S may possess a high selectivity targeting hepatocellular carcinoma cells without interfering with normal cells. A serial concentration gradient was applied to analyze the cell-binding affinity of aptamer Apt-07S to the two target cell lines, $\mathrm{HepG}_{2}(\mathrm{Kd}=194.7$ $\pm 69.8 \mathrm{nM})$ and SMMC-7721 $(\mathrm{Kd}=224.2 \pm 60.4 \mathrm{nM})$. Figure 3 clearly shows the specificity and affinity of aptamer Apt-07S to the target cancer cells.
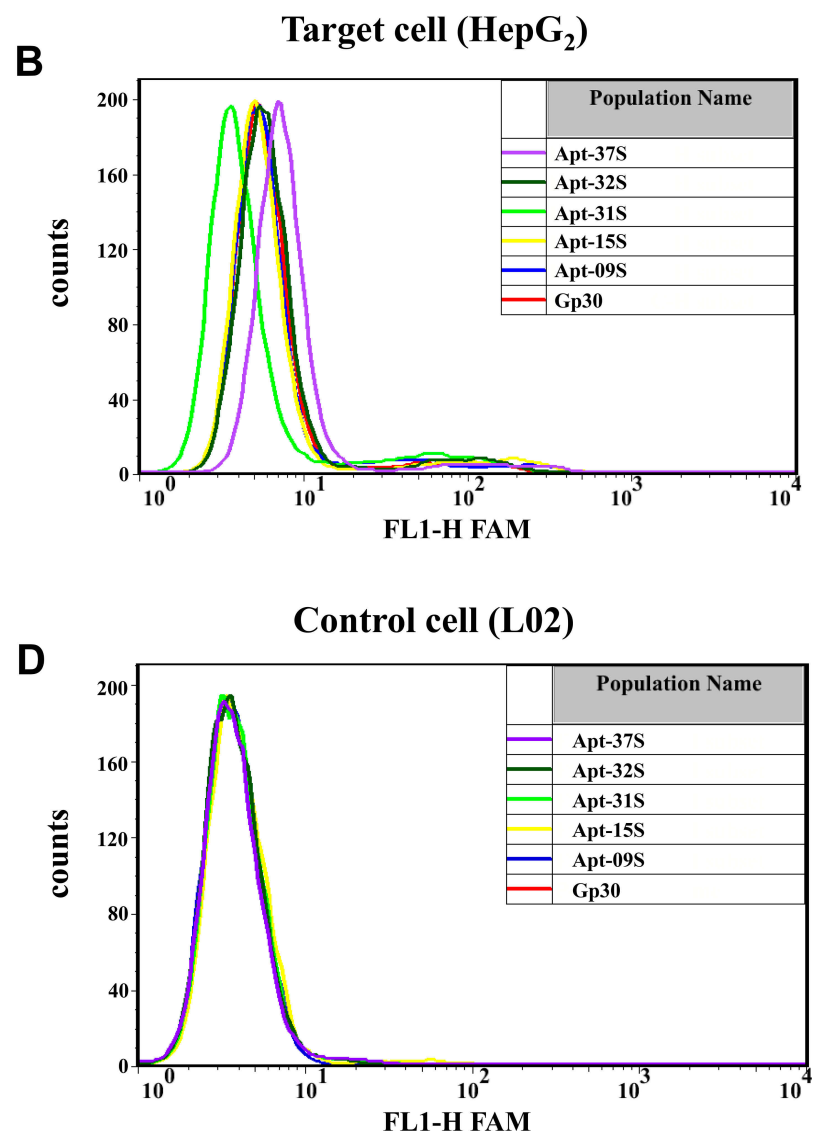

Figure 2 Primary screening of candidate aptamers based on their cell-binding abilities to $\mathrm{HepG}_{2}$ cells by applying flow cytometrics. (A,B) Fluorescence intensity analysis of the candidate aptamers binding to the target cell $\mathrm{HepG}_{2}$. (C,D) Fluorescene intensity analysis of the candidate aptamers binding to the control cell L02. Gp30 was FAM labeled initial library used as a control here. Approximately $3 \times 10^{5} \mathrm{HepG}_{2}$ cells were stained with candidate aptamers; the final concentration of each input aptamer was $500 \mathrm{nM}$. 
Table 2 Cell-Binding Abilities to Ten Kinds of Cancer Cell Lines and Normal Cells

\begin{tabular}{|l|l|l|l|}
\hline Cell Line & Cancer Type & Apt-07S & Apt-37S \\
\hline HepG 2 & Hepatocellular carcinoma (target cell) & +++ & + \\
SMMC-772I & Hepatocellular carcinoma (target cell) & +++ & ++ \\
L02 & Normal hepatocytes (control cell) & - & + \\
HCC-LM3 & Hepatocellular carcinoma & ++ & - \\
Huh.7 & Hepatocellular carcinoma & + & - \\
MCF-7 & Breast carcinoma & - & - \\
SW480 & Colon adenocarcinoma & - & + \\
HBL-100 & normal breast cell line (incorporated with SV40 virus gene) & - & + \\
H460 & Large cell lung carcinoma & + \\
MGC-803 & Gastric carcinoma & + \\
\hline
\end{tabular}

Notes: *A threshold was set based on the fluorescence intensity of cells stained with FAM-labeled initial library so that the percentage of cells with FAM-labeled aptamer above the threshold was employed to evaluate the cell-binding abilities of aptamers:,$-<10 \%$; +, $10-35 \%$; ++, 35-60\%; +++, 60-85\%; ++++, >85\%.

FAM-labeled initial library Gp30 was used as a negative control in confocal imaging observation. There was obvious fluorescence on $\mathrm{HepG}_{2}$ and SMMC-7721 cells when incubated with Apt-07S, while L02 cells displayed the opposite. By contrast, no fluorescence was observed on cells incubated with Gp30 (Figure 4). Consequently, the
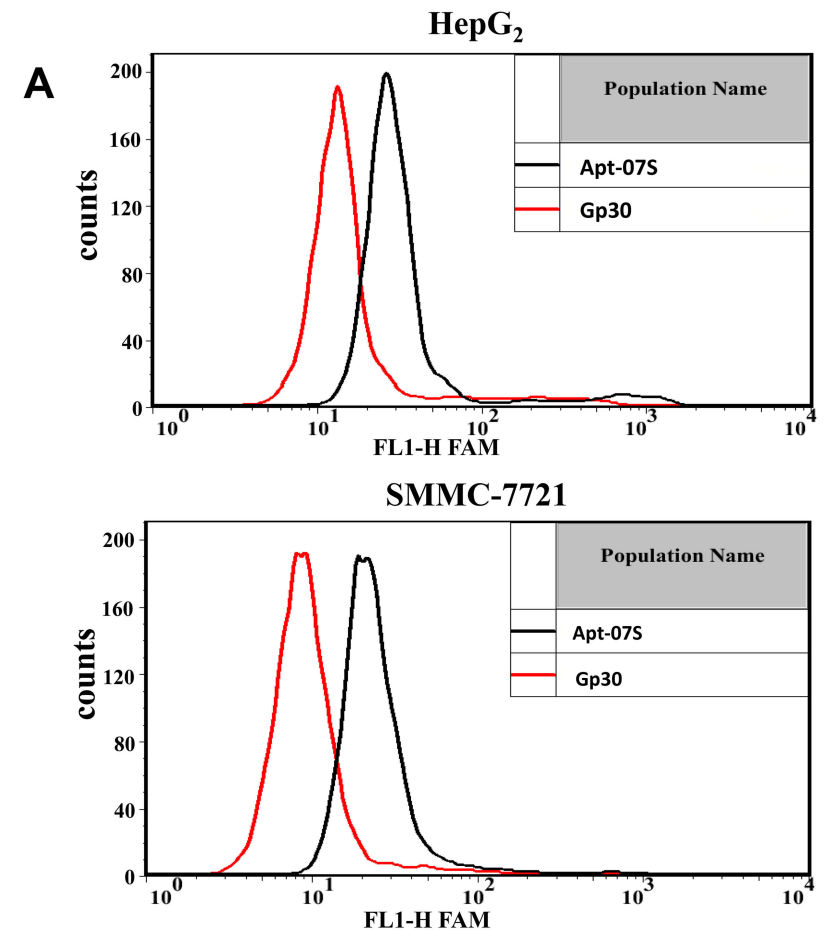

B
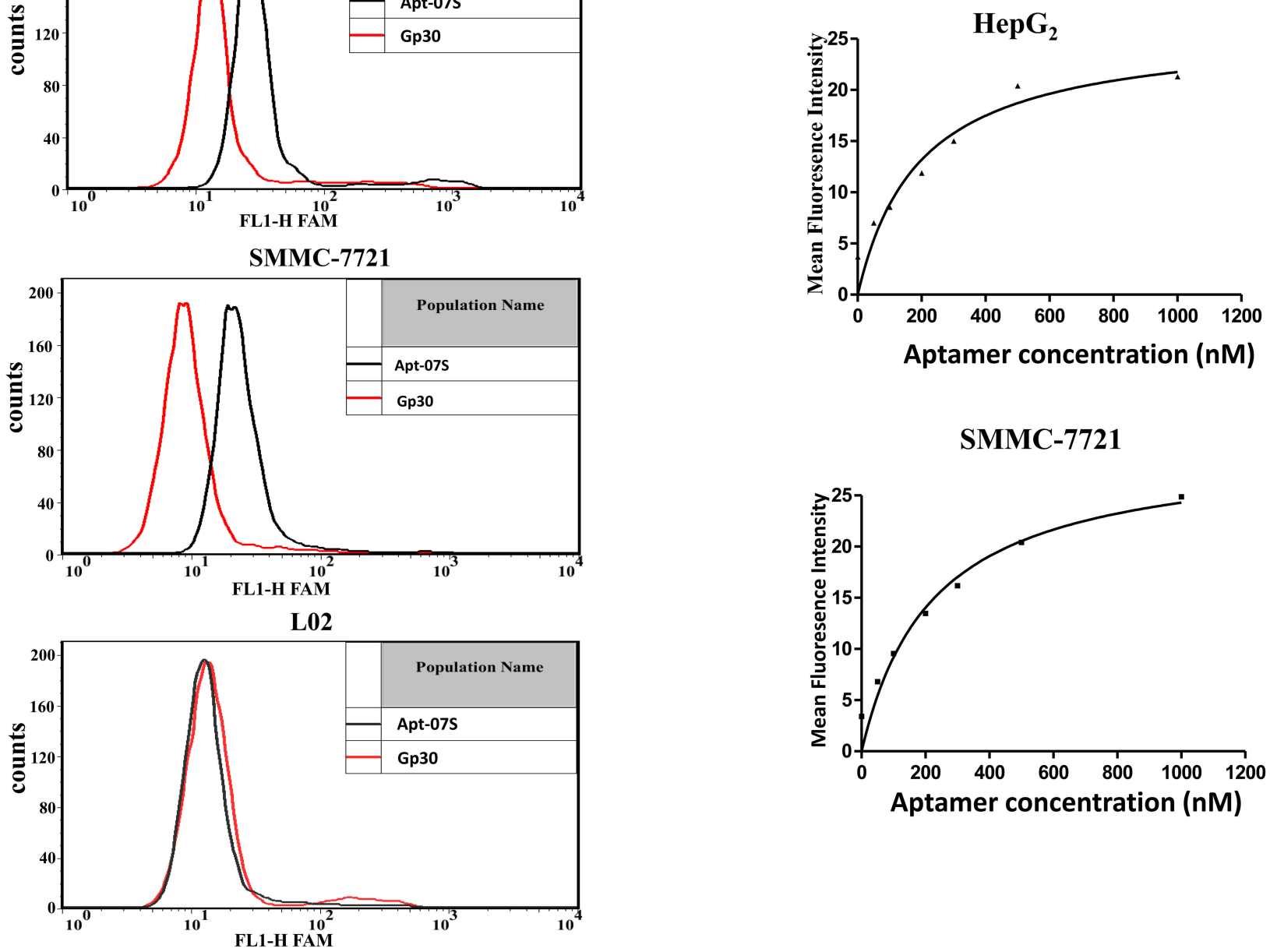

Figure 3 The specificity and binding affinity of aptamer Apt-07S. (A) Aptamer Apt-07S could specifically recognize two target cells (HepG 2 and SMMC-772I) but not negative cell L02. (B) Binding curves of aptamer Apt-07S to two target hepatocellular carcinoma cells, HepG and SMMC-772I. 
confocal imaging further confirmed the specificity of aptamer Apt-07S. Moreover, it was observed that FAM-labeled Apt-07S distributed both on the cell surface and in the cytoplasm of target cells, implying that Apt-07S could specifically recognize the target cells and may be a potential vehicle molecule in the delivery of anticancer drugs into the cancer cells.

\section{The Potential Use of Apt-07S in Delivery of Anticancer Drugs}

The secondary structures of Apt-07S and integrated ssDNA (Apt-07S-ASODN-Plk1) were predicted and plotted by RNA structure (version 6.2) software (Figure 5A and B). Cell-growth inhibition rate analysis was applied to investigate two problems: whether aptamer Apt-07S was toxic to $\mathrm{HepG}_{2}$ and SMMC-7721 cells and whether Apt-07S could increase the inhibitory effect of ASODN-Plk1 on target cells. It is clearly shown in Figure $5 \mathrm{C}$ and $\mathrm{D}$ that Apt-07S was toxic to $\mathrm{HepG}_{2}$ (above $10 \mu \mathrm{M},{ }^{*} P<0.05$ ) and SMMC-7721 (above $20 \mu \mathrm{M}, * P<0.05)$, indicating that it may be used as an anticancer drug with further modification. Compared with ASODN-Plk1, the inhibition of the integrated ssDNA (Apt-07S-ASO-Plk1) to $\mathrm{HepG}_{2}$ and SMMC-7721 cells was far more effective than ASODN-Plk1 $(\bullet \bullet, P<0.01)$. It has also been shown that the integrated ssDNA (Apt-07S-ASOPlk1) was not toxic to the control hepatocytes, L02, implying there might be few side effects when it was used as a drug.
Western blotting was performed to determine the expression of protein PLK1. As shown in Figure 5F, ASODN-Plk1 had little influence on the protein expression of gene Plk1; however, the integrated ssDNA (Apt-07S-ASO-Plk1) decreased the expression of protein PLK1 to some extent, but no obvious difference was observed among the expression of PLK1 on L02 cells.

\section{Discussion}

The aptamer can specifically bind to a variety of target molecules due to their specific three-dimensional (3D) structure formed by hairpin, internal loop, pseudoknot, bulge, and G-tetramer. ${ }^{35}$ Researchers preferred to develop RNA aptamers for their higher affinity and high diversity of 3D structure in the early stage. ${ }^{36}$

However, RNA aptamers are easily degraded by RNase, which undoubtedly increases the difficulty of its screening and application. ${ }^{37}$ By contrast, it has been gradually realized that DNA aptamer is more incomparable in structural stability and thus receives more interest than RNA aptamer. ${ }^{38}$

Gp30, the random ssDNA library used in this study, consists of a random sequence of $30 \mathrm{nt}$ in the middle with a diversity of approximately $10^{13}-10^{15}$ random ssDNA. The increase in fluorescence intensity of the library and the appearance of mono-clones with high frequency indicated the enrichment of the ssDNA library. Unexpectedly, a few ssDNA sequences seem "truncated" for some unknown reason, the length of

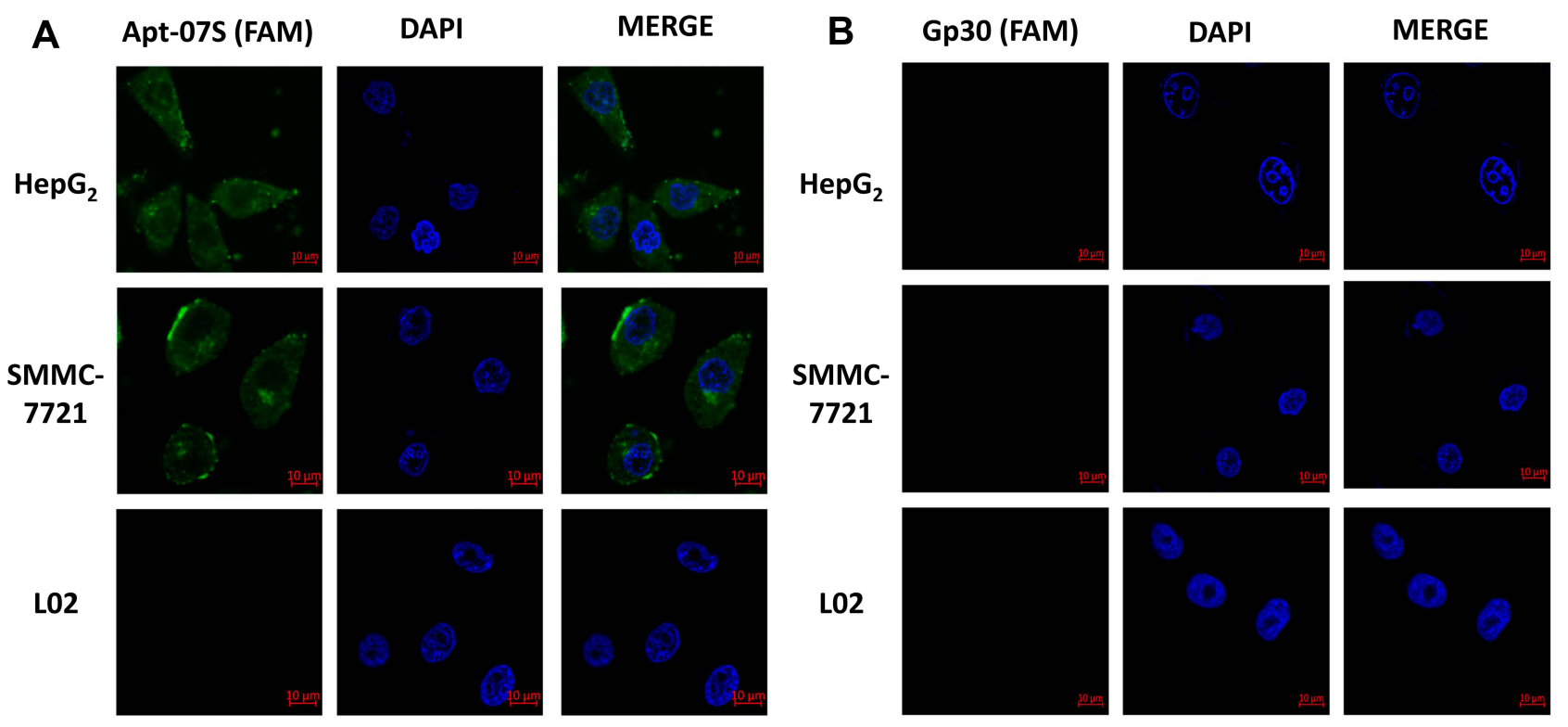

Figure 4 The binding position of aptamer Apt-07S displayed by confocal imaging. Cell nuclei were stained by DAPI (blue) and Apt-07S was labeled with FAM (green). It was shown that Apt-07S distributed both on the cell surface and in the cytoplasm of target cells. 
A

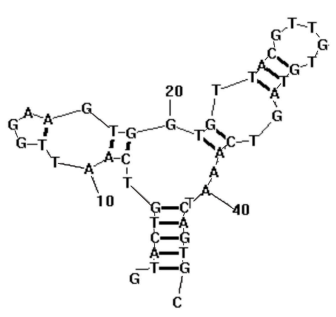

C
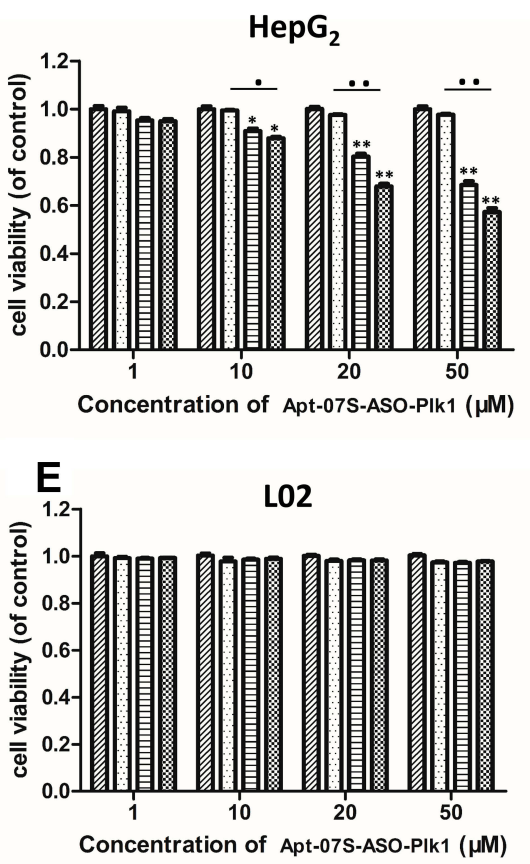

B

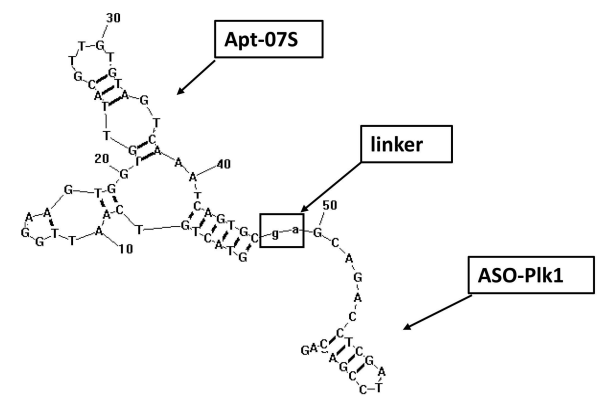

D

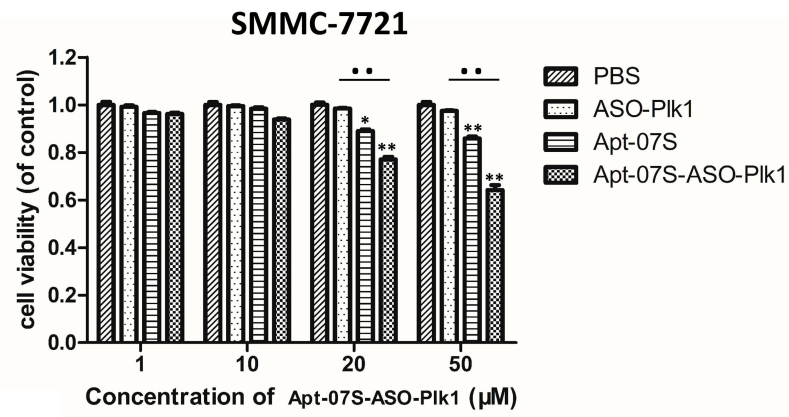

F

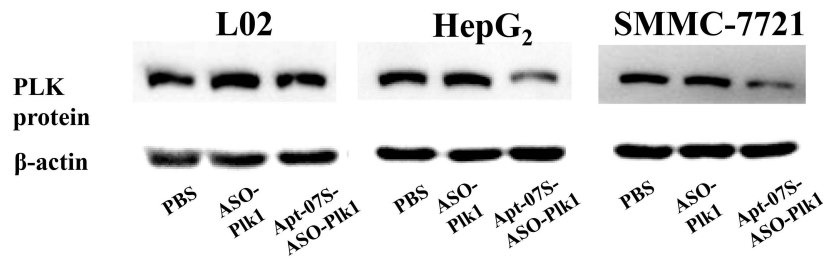

Figure 5 Aptamer Apt-07S delivering ASODN-PlkI to HepG 2 cells as vehicles. (A, B) The secondary structures of Apt-07S and the integrated ssDNA (Apt-07S-ASO-PlkI). Obviously, the connection of ASODN-PlkI did not destroy the secondary structure of Apt-07S. (C-E) $* P<0.05$, $* * P<0.0$ I, group PBS (control, cells were only treated with PBS) VS other treatment groups, respectively; $\bullet P<0.05$, $\bullet P<0.01$, ASO-PIkI VS the integrated ssDNA (Apt-07S-ASO-PIkI). (F) The expression of protein PLKI in L02, $\mathrm{HepG}_{2}$, and SMMC-772I. Cells were treated with PBS, ASO-PlkI, and the integrated ssDNA (Apt-07S-ASO-PlkI) respectively.

whose random sequences was shorter than $30 \mathrm{nt}$ as expected. To solve this problem, quite a few improvements were made, including changing PCR components, applying high-fidelity DNA polymerase, and adjusting the reaction parameters of PCR. However, similar phenomena of truncation appear. In addition, the candidate aptamer Apt-31S, with the highest abundance, could not recognize either of the two target hepatocellular cells (Figure 2). A few investigations have been conducted, and it is hypothesized that PCR amplification bias ${ }^{39}$ may decrease the efficiency of SELEX. During the process of PCR, a variety of ssDNA in the library could not be uniformly multiplied. The easily amplified candidate with a simple structure is more predominant while the enriched target binding candidate is a minority due to the complicated structure. Due to this, the "truncated" ssDNA might be some short simple ssDNA originally existing in the library, and rapidly multiplied because of their simplicity.

Despite the above problems, we successfully isolated an aptamer, Apt-07S, with double targets, which was unprecedented in previous work. In the present study, the selected aptamer could specifically recognize both of the target cell lines, $\mathrm{HepG}_{2}$ and SMMC-7721, with good affinity (Figure 3), the specificity of which was further confirmed by confocal imaging as shown in Figure 4. It is observed in Figure 4 that the FAM-labeled Apt-07S is distributed both on the cell surface and in the 
cytoplasm, suggesting that Apt-07S could specifically recognize the target and enter the cancer cell. An antisense nucleotide (ASODN-Plk1) was connected to the $3^{\prime}$ end of Apt-07S to be an integrated ssDNA (Figure 5B). Generally, it is difficult for anti-sense nucleotide to enter the cells autonomously without the carrier of transfection reagent. Thus, ASODN-Plk1 in this study show little inhibition on the growth of cells. Compared with ASODN-Plk1, the integrated ssDNA (Apt-07S-ASOPlk1) was superior in inhibiting the growth of target cells. It can be presumed that the structure of Apt-07S in the integrated ssDNA may help enhance the ability of the integrated ssDNA into cells and thus inhibit the cell growth and decrease the expression of protein PLK1. Further demonstrations are needed on the mechanism of the Apt-07S entering the cells and potential in delivering the anticancer drug.

The control and cure of liver cancer by traditional therapeutic methods have been a great challenge, due to their poor targeting, an inefficient delivery system for drugs, and severe side effects. Currently, aptamers have been applied to the study and development of a delivery system for anticancer drugs. ${ }^{40}$ The aptamer Apt-07S developed in this study can specifically recognize four kinds of HCC cell lines $\left(\mathrm{HepG}_{2}\right.$, SMMC-7721, HCCLM3, and Huh.7) but show no cell-binding ability to the normal hepatocyte L02 and normal breast cell HBL-100 (Table 2), implying that it may reduce side effects and improve the local drug concentration due to its targeting property when applied in drug delivery systems. Further modifications and optimization on the structure of Apt07S are still needed to enhance the intracellular stability and reduce its toxicity.

\section{Conclusion}

We successfully developed an aptamer, Apt-07S, via cellSELEX using two kinds of hepatocellular carcinoma cell lines, $\mathrm{HepG}_{2}$ and SMMC-7721. Apt-07S can recognize four kinds of hepatocellular carcinoma cells and shows little cell-binding ability to normal cells and four cell lines of different cancer types, revealing a high specificity for hepatocellular carcinoma cells. It was preliminarily verified that Apt-07S might be a targeting agent for the delivery of anticancer drugs.

\section{Acknowledgment}

This work was supported by Shandong Province Science Funding (2014GGH218023).

\section{Disclosure}

Yin-Lin Ge, Xiao-Xia Yu and Ke-Li Ge have a patent (201810082398.6) pending. The authors report no other conflicts of interest in this work.

\section{References}

1. Bray F, Ferlay J, Soerjomataram I, Siegel RL, Torre LA, Jemal A. Global cancer statistics 2018: GLOBOCAN estimates of incidence and mortality worldwide for 36 cancers in 185 countries. CA Cancer J Clin. 2018;68(6):394-424. doi:10.3322/caac.21492

2. Llovet JM, Zucman-Rossi J, Pikarsky E, et al. Hepatocellular carcinoma. Nat Rev Dis Primers. 2016;2(1):16018. doi:10.1038/nrdp.2016.18

3. Llovet JM. Updated treatment approach to hepatocellular carcinoma. $J$ Gastroenterol. 2005;40(3):225-235. doi:10.1007/s00535-005-1566-3

4. Vogel A, Cervantes A, Chau I, et al. Hepatocellular carcinoma: ESMO clinical practice guidelines for diagnosis, treatment and follow-up. Ann Oncol. 2019;30(5):871-873. doi:10.1093/annonc/mdy510

5. Llovet JM, Ricci S, Mazzaferro V, et al. Sorafenib in advanced hepatocellular carcinoma. $N$ Engl J Med. 2008;359(4):378-390. doi:10.1056/NEJMoa0708857

6. Ellington AD, Szostak JW. In vitro selection of RNA molecules that bind specific ligands. Nature. 1990;346(6287):818-822. doi:10.1038/ $346818 \mathrm{a} 0$

7. Tuerk C, Gold L. Systematic evolution of ligands by exponential enrichment: RNA ligands to bacteriophage T4 DNA polymerase. Science. 1990;249(4968):505-510. doi:10.1126/science.2200121

8. Shangguan D, Meng L, Cao ZC, et al. Identification of liver cancerspecific aptamers using whole live cells. Anal Chem. 2008;80 (3):721-728. doi:10.1021/ac701962v

9. Shangguan D, Cao Z, Meng L, et al. Cell-specific aptamer probes for membrane protein elucidation in cancer cells. J Proteome Res. 2008;7 (5):2133-2139. doi:10.1021/pr700894d

10. Chang YM, Donovan MJ, Tan WH. Using aptamers for cancer biomarker discovery. J Nucleic Acids. 2013;2013:1-7. doi:10.1155/ 2013/817350

11. Kaur H, Li JJ, Bay BH, Yung LY. Investigating the antiproliferative activity of high affinity DNA aptamer on cancer cells. PLoS One. 2013;8(1):e50964. doi:10.1371/journal.pone.0050964

12. Kaur H. Recent developments in cell-SELEX technology for aptamer selection. Biochimica Et Biophysica Acta (BBA) - General Subjects. 2018;1862(10):2323-2329. doi:10.1016/j.bbagen.2018.07.029

13. Wang YY, Luo Y, Bing T, et al. DNA aptamer evolved by cell-selex for recognition of prostate cancer. PLoS One. 2014;9(6):e100243. doi:10.1371/journal.pone. 0100243

14. Lee JF, Stovall GM, Ellington AD. Aptamer therapeutics advance. Curr Opin Chem Biol. 2006;10(3):282-289. doi:10.1016/j.cbpa.2006.03.015

15. Li S, Wang W, Ding H, et al. Aptamer BC15 against heterogeneous nuclear ribonucleoprotein $\mathrm{A} 1$ has potential value in diagnosis and therapy of hepatocarcinoma. Nucleic Acid Ther. 2012;22(6):391-398. doi:10.1089/nat.2012.0363

16. Xu J, Teng IT, Zhang L, et al. Molecular recognition of human liver cancer cells using DNA aptamers generated via cell-SELEX. PLoS One. 2015;10(5):e0125863. doi:10.1371/journal.pone.0125863

17. Zhao Z, Xu L, Shi X, Tan W, Fang X, Shangguan D. Recognition of subtype non-small cell lung cancer by DNA aptamers selected from living cells. Analyst. 2009;134(9):1808-1814. doi:10.1039/b904476k

18. Kaur H, Bruno JG, Kumar A, Sharma TK. Aptamers in the therapeutics and diagnostics pipelines. Theranostics. 2018;8(15):40164032. doi: $10.7150 /$ thno. 25958

19. Liu N, Zhou C, Zhao J, Chen YJCI. Reversal of paclitaxel resistance in epithelial ovarian carcinoma cells by a MUC1 aptamer-let-7i chimera. Cancer Invest. 2012;30(8):577-582. doi:10.3109/073579 07.2012.707265 
20. Luo S, Wang S, Na L, Chen F, Hu C, Zhang K. The application of aptamer 5TR1 in triple negative breast cancer target therapy. $J$ Cell Biochem. 2018;119(1):896-908. doi:10.1002/jcb.26254

21. Zhou JH, Rossi J. Aptamers as targeted therapeutics: current potential and challenges. Nat Rev Drug Discov. 2017;16(3):181-202. doi:10.10 38/nrd.2017.86

22. Ninomiya K, Kaneda K, Kawashima S, Miyachi Y, Ogino C, Shimizu N. Cell-SELEX based selection and characterization of DNA aptamer recognizing human hepatocarcinoma. Bioorg Med Chem Lett. 2013; 23(6):1797-1802. doi:10.1016/j.bmcl.2013.01.040

23. Yu G, Li H, Yang S, Wen J, Niu J, Zu Y. ssDNA aptamer specifically targets and selectively delivers cytotoxic drug doxorubicin to HepG2 cells. PLoS One. 2016;11(1):e0147674. doi:10.1371/journal.pone.0147674

24. Lu B, Wang J, Zhang J, et al. Screening and verification of ssDNA aptamers targeting human hepatocellular carcinoma. Acta Biochim Biophys Sin. 2014;46(2):128-135. doi:10.1093/abbs/gmt130

25. Kaur H, Yung LY. Probing high affinity sequences of DNA aptamer against VEGF165. PLoS One. 2012;7(2):e31196. doi:10.1371/journal.pone.0031196

26. Chen H, Yuan $\mathrm{CH}$, Yang YF, et al. Subtractive cell-SELEX selection of DNA aptamers binding specifically and selectively to hepatocellular carcinoma cells with high metastatic potential. Biomed Res Int 2016:1-9. doi:10.1155/2016/5735869

27. Meng L, Yang L, Zhao X, et al. Targeted delivery of chemotherapy agents using a liver cancer-specific aptamer. PLoS One. 2012;7(4): e33434. doi:10.1371/journal.pone.0033434

28. Hu Z, He J, Gong W, et al. TLS11a aptamer/CD3 antibody anti-tumor system for liver cancer. J Biomed Nanotechnol. 2018;14(9):16451653. doi:10.1166/jbn.2018.2619

29. Wang R, Zhao N, Li S, et al. MicroRNA-195 suppresses angiogenesis and metastasis of hepatocellular carcinoma by inhibiting the expression of VEGF, VAV2, and CDC42. Hepatology. 2013;58(2):642-653. doi:10.1002/hep. 26373

30. Yin S, Li J, Hu C, et al. CD133 positive hepatocellular carcinoma cells possess high capacity for tumorigenicity. Int J Cancer. 2007;120 (7):1444-1450. doi:10.1002/ijc.22476
31. Tao KS, Dou KF, Wu XA. Expression of angiostatin cDNA in human hepatocellular carcinoma cell line SMMC-7721 and its effect on implanted carcinoma in nude mice. World J Gastroenterol. 2004;10 (10):1421-1424. doi:10.3748/wjg.v10.i10.1421

32. Jiang Y, Zhou X-D, Liu Y-K, et al. Antisense Tcf inhibits the neoplastic growth of liver cancer cells. J Cancer Res Clin Oncol. 2004;130(11):671-678. doi:10.1007/s00432-004-0580-9

33. Elez R, Piiper A, Kronenberger B, et al. Tumor regression by combination antisense therapy against Plk1 and Bcl-2. Oncogene. 2003;22(1):69-80. doi:10.1038/sj.onc. 1206038

34. Cao X, Li S, Chen L, et al. Combining use of a panel of ssDNA aptamers in the detection of Staphylococcus aureus. Nucleic Acids Res. 2009;37(14):4621-4628. doi:10.1093/nar/gkp489

35. Perez-Gonzalez C, Lafontaine DA, Penedo JC. Fluorescence-based strategies to investigate the structure and dynamics of aptamer-ligand complexes. Front Chem. 2016;4:33. doi:10.3389/fchem.2016.00033

36. Zhang Y, Lai BS, Juhas M. Recent advances in aptamer discovery and applications. Molecules. 2019;24(5). doi:10.3390/molecules24 050941

37. Zhang GQ, Zhong LP, Yang N, Zhao YX. Screening of aptamers and their potential application in targeted diagnosis and therapy of liver cancer. World J Gastroenterol. 2019;25(26):3359-3369. doi:10.3748/ wjg.v25.i26.3359

38. Wang T, Chen C, Larcher LM, Barrero RA, Veedu RN. Three decades of nucleic acid aptamer technologies: lessons learned, progress and opportunities on aptamer development. Biotechnol Adv. 2019;37(1):28-50. doi:10.1016/j.biotechadv.2018.11.001

39. Tsuji S, Hirabayashi N, Kato S, et al. Effective isolation of RNA aptamer through suppression of PCR bias. Biochem Biophys Res Commun. 2009;386(1):223-226. doi:10.1016/j.bbrc.2009.06.013

40. Soldevilla MM, Meraviglia-crivelli de Caso D, Menon AP, Pastor F. Aptamer-iRNAs as therapeutics for cancer treatment. Pharmaceuticals. 2018;11(4):108. doi:10.3390/ph11040108
Drug Design, Development and Therapy

\section{Publish your work in this journal}

Drug Design, Development and Therapy is an international, peerreviewed open-access journal that spans the spectrum of drug design and development through to clinical applications. Clinical outcomes, patient safety, and programs for the development and effective, safe, and sustained use of medicines are a feature of the journal, which has also been accepted for indexing on PubMed Central. The manuscript management system is completely online and includes a very quick and fair peer-review system, which is all easy to use. Visit http://www. dovepress.com/testimonials.php to read real quotes from published authors. 